$S$ and the integrals of the second member are taken along the arcs $s^{(v)}$ of the spirals $\left(S_{a}^{\nu}\right)$ from $\left(2^{\prime \prime}\right)$. The proof, proceeding along the same lines as that of (6), is suppressed here.

REMARK. The explicit values of the elementary integrals (3) are, of course, well known; but we refrain purposely from using them, as they are not needed. It is, indeed, sufficient for our proofs to know that those integrals depend only on the exponents $m, n$ and are independent of $\phi, \theta$, or $r$.

UNIVERSITY OF SASKATCHEWAN

\title{
ON THE DENSITY THEOREM
}

\section{A. PAPOULIS}

1. Introduction. Let $F$ be a set on the plane and $x$ a point of $F$. With $\left\{I_{n}\right\}$ an arbitrary sequence of intervals ${ }^{1}$ containing the point $x$ and with diameter tending to zero, we form the sequence $\left|F \cdot I_{n}\right| /\left|I_{n}\right| .^{2}$ It has been shown (see [1] and [2]) ${ }^{3}$ that for almost ${ }^{4}$ all points $x$ of $F$,

$$
\lim _{I_{n}} \frac{\left|F \cdot I_{n}\right|}{\left|I_{n}\right|}=1 .
$$

If the sequence $\left\{I_{n}\right\}$ of intervals is replaced by a sequence of arbitrary rectangles with sides not necessarily parallel to the axes of coordinates, then the above ceases to be true. H. Busemann and W. Feller (see [1]) have shown that if the direction of some one of the sides of the rectangles $\left\{I_{n}\right\}$ varies within any nonzero angle, then (1) is no longer true for all sets $F$.

The purpose of the following is to show that even if the direction of the rectangles $\left\{I_{n}\right\}$ converging to the point $x$ is fixed, then (1) is still not true for some sets, provided of course that the fixed direction may vary from point to point.

Received by the editors October 17, 1950.

${ }^{1}$ Rectangles with sides parallel to the coordinate axes.

${ }^{2}$ The number $|E|$ will mean the two-dimensional Lebesgue-measure of the set $E$.

${ }^{3}$ Numbers in brackets refer to the references at the end of the paper.

${ }^{4}$ By "almost all points $x$ of a set $E^{n}$ we shall mean all points of $E$ except for a set of measure zero; this will also be indicated by p.p. 
2. Part A. We shall construct ${ }^{5}$ a set $E$ included in the unit square $Q$ and such that:

(2A) $|E|<3 \epsilon$, where $\epsilon$ is an arbitrary number; given $x \in Q$ there exists a sequence $\left\{I_{n}\right\}$ of rectangles containing the point $x$, with diameter tending to zero, and such that:

$$
\lim \sup _{I_{n}} \frac{\left|E \cdot I_{n}\right|}{\left|I_{n}\right|}>\frac{1}{3} \text {. }
$$

This construction will be given in $\$ \$ 3-7$.

3. Definition. Given a plane set $E$, let $M(E)$ be the set of points $x$ of the plane with the property that there exists a line-segment $s$ containing $x$ and such that

$$
\frac{m_{1}(E \cdot s)}{m_{1}(s)}>\frac{1}{2} \text {. }
$$

If $E$ is a circle of radius $R$, then clearly $M(E)$ is a concentric circle of radius $3 R$.

\section{Lemma. $^{7}$}

$$
\underset{E}{\text { g.l.b. }} \frac{|E|}{|M(E)|}=0
$$

as $E$ ranges over all plane sets.

If $E$ is a circle, then $|E| /|M(E)|=1 / 9$; if $E$ is a triangle, then $|E| /|M(E)|=1 / 13$.

Proof. It suffices to show that given an $\epsilon$ we can construct a set $E$ such that

$$
\frac{|E|}{|M(E)|}<\epsilon
$$

Consider the open isosceles triangle $S_{0}=A B C$ of base $B C=a$, angle $B A C=\theta$, and altitude $h$, with an axis $\alpha-\alpha$ parallel to the $y$-axis. Clearly the points of the trapezoid $T_{0}=B C E D$, where $D E$ is the line $y=-h$, belong to $M\left(S_{0}\right)$. Draw the lines $y=h-h^{\prime}$ and $y=h+h^{\prime}$ [1].

- This construction is similar to the one given by H. Busemann and W. Feller in

6 The number $m_{1}(s)$ will mean the linear measure of the set $s$.

7 The above lemma is based on a construction used by $O$. Perron in giving a simple solution to the Besicovitch-Kakeya problem; it was utilized also by $\mathrm{H}$. Busemann and W. Feller in their treatment of the density theorem. 
( $h^{\prime}$ to be determined soon); they intersect $A B$ and $A C$ and their extensions at the points $K, L$ and $L^{\prime}, K^{\prime}$; the lines $K K^{\prime}$ and $L L^{\prime}$ intersect $B C$ at the points $M$ and $N$. The open figure $S_{1}$ $=A K^{\prime} K B C L L^{\prime} A$ has an area $\left|S_{1}\right|=\left|S_{0}\right|\left\{1+2\left(h^{\prime} / h\right)^{2}\right\}$; the line $y=-\left(h+h^{\prime}\right)$ intersects the extensions of $A B$ and $A C$ at the points $D_{1}$ and $E_{1}$ forming the trapezoid $T_{1}=B C E_{1} D_{1}$; clearly $\left|T_{1}\right|$ $>\left|T_{0}\right|\left(1+h^{\prime} / h\right)$ and $T_{1} \subset M\left(S_{1}\right)$.

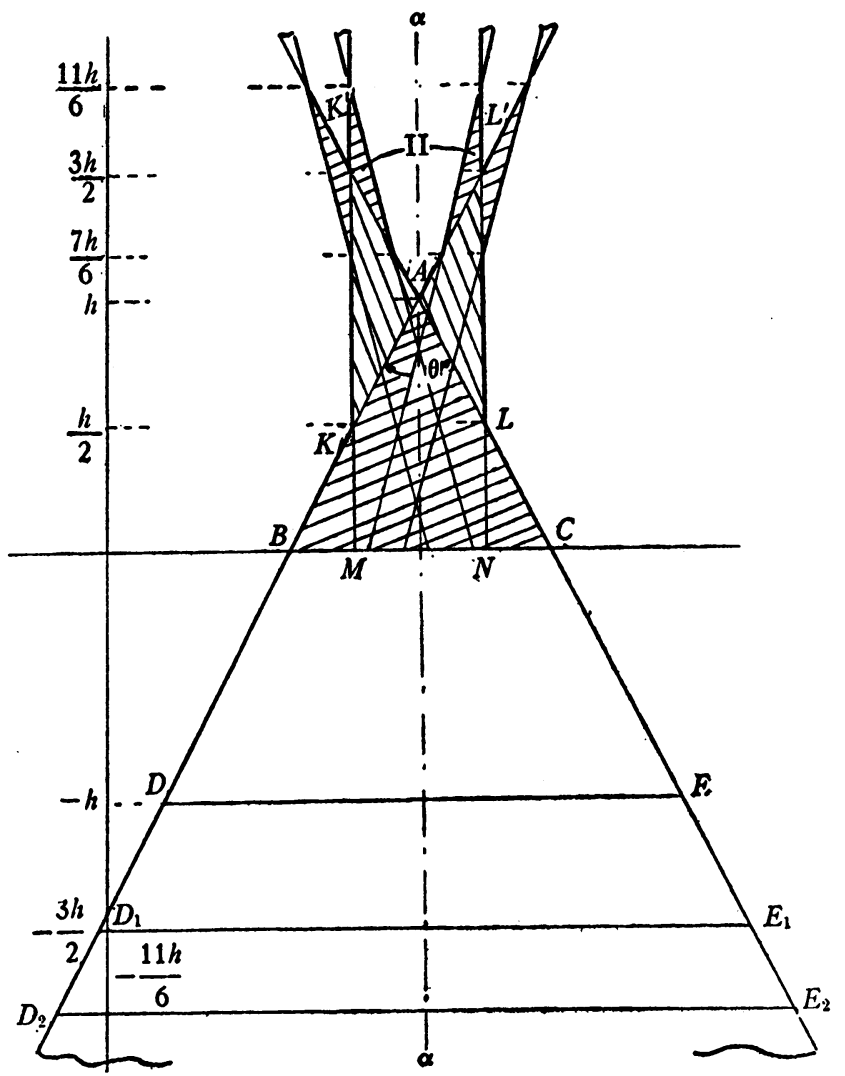

FIG. 1

With $h^{\prime}=h / 2$ we have

$$
\left|S_{1}\right|=\left|S_{0}\right|\left\{1+2(1 / 2)^{2}\right\}, \quad\left|T_{1}\right|>\left|T_{0}\right|\{1+1 / 2\} .
$$

We repeat the above construction with each of the triangles $K^{\prime} M C$ and $L^{\prime} B N$ with $h^{\prime}=h / 3$; we thus obtain the open figure $S_{2}$ consisting of $S_{1}$ and the four added triangles II, and the trapezoid $T_{2}=B C E_{2} D_{2}$. Again 


$$
\begin{aligned}
T_{2} & \subset M\left(S_{2}\right), \\
\left|S_{2}\right| & =\left|S_{0}\right|\left\{1+2(1 / 2)^{2}+2(1 / 3)^{2}\right\}, \\
\left|T_{2}\right| & >\left|T_{0}\right|\{1+1 / 2+1 / 3\} .
\end{aligned}
$$

Repeating the above process on each of the four new triangles, whose base is part of $B C$, with $h^{\prime}=h / 4$, we obtain the open figure $S_{3}$, consisting of $S_{2}$ and the eight added triangles, and the trapezoid $T_{3}=B C E_{3} D_{3} ;$ as before

$$
\begin{aligned}
T_{3} & \subset M\left(S_{3}\right), \\
\left|S_{3}\right| & =\left|S_{0}\right|\left\{1+2(1 / 2)^{2}+2(1 / 3)^{2}+2(1 / 4)^{2}\right\}, \\
\left|T_{3}\right| & >\left|T_{0}\right|\{1+1 / 2+1 / 3+1 / 4\} .
\end{aligned}
$$

At the $n$th such step with $h^{\prime}=h / n$, we obtain the open figure $S_{n}$ and the trapezoid $T_{n}=B C E_{n} D_{n}$; they satisfy the relation

$$
\begin{aligned}
T_{n} & \subset M\left(S_{n}\right), \\
\left|S_{n}\right| & =\left|S_{0}\right|\left\{1+2(1 / 2)^{2}+\cdots+2(1 / n)^{2}\right\}, \\
\left|T_{n}\right| & >\left|T_{0}\right|\{1+1 / 2+\cdots+1 / n\} .
\end{aligned}
$$

Since $1+1 / 2+\cdots+1 / n+\cdots$ diverges, $1+(1 / 2)^{2}+\cdots$ $+(1 / n)^{2}+\cdots$ converges, and $\left|T_{0}\right| \neq 0$, it follows that for a certain $n=N(\epsilon)$ we shall have

$$
\frac{\left|S_{N}\right|}{\left|S_{N}+T_{N}\right|}<\epsilon,
$$

and since $S_{N}+T_{N} \subset M\left(S_{N}\right)$, (4) has been established and the lemma is proved.

5. The set $S_{N}+T_{N}$ will be denoted by $B(\epsilon ; \theta)$, where $\epsilon$ and $\theta$ are as in the previous section, and the set $S_{N}$ by $S(\epsilon ; \theta)$. Thus

$$
\frac{|S(\epsilon ; \theta)|}{|B(\epsilon ; \theta)|}<\epsilon, \quad B(\epsilon ; \theta) \subset M(S(\epsilon ; \theta)) .
$$

REMARK 1 . Obviously $\epsilon$ and $\theta$ can be taken arbitrarily small in the above construction.

6. Construction of $E$. In this section $\theta$ will be kept constant and will be omitted in the expressions $S(\epsilon ; \theta)$ and $B(\epsilon ; \theta)$.

First step. By $[B(\epsilon)]$ we shall mean the class of all sets similar to $B(\epsilon)$ and similarly placed, with diameter smaller than one. For every $x \in Q$, there exists a sequence of sets belonging to $[B(\epsilon)]$, containing the point $x$ and with diameter tending to zero, hence (Vitali covering 
theorem) there exists a sequence $\{B(\epsilon)\}$ of disjoint sets belonging to $[B(\epsilon)]$ and covering $Q$ p.p. The sets $S(\epsilon)$ which are parts of $B(\epsilon)$ of the sequence $\{B(\epsilon)\}$ form another sequence $\{S(\epsilon)\}$ whose sum we denote by $E_{0}$; from (8) and the disjointness of the sets $B(\epsilon)$ of $\{B(\epsilon)\}$ we conclude that

$$
\left|E_{0}\right|<\epsilon|Q|=\epsilon .
$$

Second step. By $[B(\epsilon / 2)]$ we shall mean the class of all sets similar to $B(\epsilon / 2)$ and similarly placed with diameter less than $1 / 2$. As before there exists a sequence $\{B(\epsilon / 2)\}$ of disjoint sets belonging to $[B(\epsilon / 2)]$ and covering $Q$ p.p.; the sets $S(\epsilon / 2)$ which are parts of $B(\epsilon / 2)$ of the sequence $\{B(\epsilon / 2)\}$ form another sequence $\{S(\epsilon / 2)\}$ whose sum we denote by $E_{1}$; as before,

$$
\left|E_{1}\right|<\frac{\epsilon}{2}|Q|=\epsilon / 2^{k}
$$

$(k+1)$ th step. By $\left[B\left(\epsilon / 2^{k}\right)\right]$ we shall mean the class of all sets similar to $B\left(\epsilon / 2^{k}\right)$ and similarly placed, with diameter smaller than $2 / 2^{k}$. Again there exists a sequence $\left\{B\left(\epsilon / 2^{k}\right)\right\}$ of disjoint sets belonging to $\left[B\left(\epsilon / 2^{k}\right)\right]$ and covering $Q$ p.p., and the sequence $\left\{S\left(\epsilon / 2^{k}\right)\right\}$ of the $S\left(\epsilon / 2^{k}\right)$ sets whose sum we denote by $E_{k}$; as before

$$
\left|E_{k}\right|<\epsilon / 2^{k}
$$

and so we continue.

Since we repeat the process an enumerable number of times, the set of points of $Q$ which are not covered at least once in this process has a zero measure; hence it can be covered by an open set $G$ of area smaller than $\epsilon$; with $E=G+\sum_{k=0}^{\infty} E_{k}$, we have

$$
|E| \leqq|G|+\sum_{k=0}^{\infty}\left|E_{k}\right|<\epsilon+\sum_{k=0}^{\infty} \frac{\epsilon}{2^{k}}=3 \epsilon .
$$

7. We shall now show that $E$ satisfies the requirements of $\S 2$. Suppose $x \in Q$; if $x$ belongs to $E, E$ being open, we can cover $x$ with a circle $C_{x} \subset E$. If we take any sequence $\left\{I_{n}\right\}$ of intervals included in $C_{x}$ and with diameter tending to zero, we have $\left|E \cdot I_{n}\right| /\left|I_{n}\right|=1>1 / 3$; hence (2A) is true. Suppose $x$ is not a point of $E$; it suffices to show that given $\delta>0$ there exists an interval $I_{k}$ of diameter smaller than $\delta$ and such that

$$
x \in I_{k}, \quad \frac{\left|E \cdot I_{k}\right|}{\left|I_{k}\right|}>\frac{1}{3}
$$


Take $k$ such that $1 / 2^{k}<\delta$; since $x \notin E$ it follows that $x \notin G$, hence it is covered by one set $B\left(\epsilon / 2^{k}\right)$ belonging to the sequence $\left\{B\left(\epsilon / 2^{k}\right)\right\}$ of the $(k+1)$ th step. From the way these sets have been constructed, it follows that there exists a line segment $s$ such that

$$
x \in s, \quad s \subset B\left(\frac{\epsilon}{2^{k}}\right), \quad \frac{m_{1}\left(s \cdot S\left(\epsilon / 2^{k}\right)\right)}{m_{1}(s)}>\frac{1}{2}
$$

where $S\left(\epsilon / 2^{k}\right)$ is the set contained in $B\left(\epsilon / 2^{k}\right)$. Since the maximum diameter of the sets $\left\{B\left(\epsilon / 2^{k}\right)\right\}$ is smaller than $1 / 2^{k}$, and $s \subset B\left(\epsilon / 2^{k}\right)$, we conclude that $d s<1 / 2^{k}{ }^{8}$ But $S\left(\epsilon / 2^{k}\right) \subset E_{k} \subset E$, hence also

$$
\frac{m_{1}(s \cdot E)}{m_{1}(s)}>\frac{1}{2}
$$

and since $E$ is open, we can find an interval $I_{k}$ containing $s$ and satisfying (15); and so our contention is proved.

REMARK 2. Since the orientation of the $I_{k}$ 's can be taken within the angle $\theta$, and since $\theta$ can be chosen arbitrarily small, it follows that the rectangles of the sequence $\left\{I_{k}\right\}$ in (2A) can be chosen with an orientation within an arbitrarily small angle.

8. With $F=Q-E$ we have from (2A) with $x \in F \subset Q$

$$
\lim _{I_{n}} \inf \frac{\left|F \cdot I_{n}\right|}{\left|I_{n}\right|}<\frac{2}{3} .
$$

Hence (1) is not true because now we have a sequence $\left\{I_{n}\right\}$ of rectangles and not of intervals.

9. Part B. We shall construct a set $E$ included in the unit square $Q$ and such that:

(2B) $|E|<5 \epsilon$ where $\epsilon$ is an arbitrary number; given $x \in Q$ there exists a sequence $\left\{I_{n}\right\}$ of rectangles of "fixed orientation," containing the point $x$, with diameter tending to zero, and such that

$$
\underset{I_{n}}{\lim \sup } \frac{\left|E \cdot I_{n}\right|}{\left|I_{n}\right|}>\frac{1}{3} \text {. }
$$

10. Notations. Suppose angle $B A C=\theta_{1}$, angle $E D F=\theta_{2}$, and $\gamma$ a given direction; if from $A$ we draw a line parallel to $\gamma$ and it falls inside $B A C$, this will be indicated by writing $\gamma \subset \theta_{1}$. If from $A$ we draw two lines parallel to $D E$ and $D F$ respectively and both fall inside $B A C$, this will be indicated by writing $\theta_{2} \subset \theta_{1}$. By $s_{\gamma}$ we shall mean a line segment parallel to the direction $\gamma$.

\footnotetext{
$d s$ will mean the diameter of the set $s$.
} 
11. Outline of the method. In the construction of Part A the direction of the line segments satisfying (14), and hence of the corresponding rectangles $I_{k}$, cannot be the same for every $k$ for the following reason: for a given $x \in Q$ the segments $s$ satisfying (14) for $k=0$ may have any direction $\gamma \subset \delta^{0}$; for $k=1$ may have any direction $\gamma \subset \delta^{1}$, for any $k$ a direction $\gamma \subset \delta^{k}$; the angle $\delta^{k}$ depends on the location of $x$ inside $B\left(\epsilon / 2^{k} ; \theta\right)$. It is possible that there is no direction $\gamma \subset \delta^{k}$ for every $k$. If we modify our second covering in such a manner that the covering sets $B\left(\epsilon / 2 ; \theta^{\prime}\right)$ have an angle $\theta^{1} \subset \delta^{0}$, then all the directions which satisfy (14) for $k=1$ must be included in $\theta^{1}$, hence in $\delta^{0}$, they therefore must satisfy (14) also for $k=0$; these possible directions form a nonzero angle $\theta^{2}$ which is used for the following step. Thus for each point $x$ we have a sequence of closed angles $\theta \supset \theta^{1}$ $\supset \theta^{2} \supset \ldots$, hence there exists a $\gamma \subset \theta^{k}$ and a segment $s_{\gamma}$ satisfying (14) for every $k$; and that is our objective.

12. We now come to our construction.

First step. We begin as in $\$ 6$; we thus have the sequences $\{B(\epsilon ; \theta)\},\{S(\epsilon ; \theta)\}$ and the set $E_{1}$ with

$$
\left|E_{1}\right|<\epsilon \text {. }
$$

For almost every $x \in Q$ there exists an $s_{\gamma_{0}}$ of direction $\gamma_{0}$ and such that

$$
x \in s, \quad d s<1, \quad \frac{m_{1}\left(s \cdot E_{1}\right)}{m_{1}(s)}>\frac{1}{2} .
$$

Since $E_{1}$ is open, it follows that all the possible directions $\gamma$ obtained by continuous rotation of $\gamma_{0}$, and for which the above is true, form a nonzero angle $w(x)$ depending on $x$. Consider the points $x$ of $Q$ such that $w(x)>\alpha$ where $\alpha$ is a given angle; they form a set $P_{\alpha}$; since to almost every $x$ of $Q$ there corresponds a nonzero angle $w(x), \lim P_{\alpha}$ as $\alpha$ tends to zero must include almost all points of $Q$. Therefore given an $\epsilon$ there exists an $\alpha_{1}$ such that

$$
\left|Q-P_{\alpha_{1}}\right|<\epsilon
$$

The points of $Q-P_{\alpha_{1}}$ can be included in an open set $G_{0}$ of measure less than $\epsilon$; with $R_{1}=E_{1}+G_{1}$ we have

$$
\left|R_{1}\right|<\epsilon+\epsilon=2 \epsilon \text {. }
$$

Consider all points $x$ of $Q$ such that for every $\gamma \subset \theta$ there exists an $s$ such that (16) is true; they form a set which we denote by $1 A_{0}^{1}$; clearly all points of $E_{1}$ belong to $1 A_{0}^{1}$ since their corresponding $w$ equals $2 \pi$. 
In the following, angles will be considered with their orientation; thus when we speak of the set $B(\epsilon ; \phi)$ we shall mean a set as in $\$ 5$ but with its axis $\alpha-\alpha$ not parallel to the $y$-axis but to the bisector of the angle $\phi$.

If we bisect $\theta$, we obtain the angles $\theta_{1}^{1}$ and $\theta_{1}^{2}$. We denote by $1 A_{1}^{t}$ $(t=1,2)$ the set of points of $Q-1 A_{0}^{1}$ such that for every $\gamma \subset \theta_{1}^{t}$ $(t=1,2)$ there exists an $s_{\gamma}$ satisfying $(16)$. We continue as follows:

We divide $\theta$ into $2^{n}(n=2,3, \cdots)$ equal angles $\theta_{n}^{t}\left(t=1,2, \cdots, 2^{n}\right)$ and denote by $1 A_{n}^{z}\left(t=1, \cdots, 2^{n}\right)$ the set of points of $Q$ $-\left\{1 A_{0}^{1}+1 A_{1}^{1}+1 A_{1}^{2}+\cdots+1 A_{n-1}^{1}+\cdots+1 A_{n-1}^{2^{n-1}}\right\}$ with the property that for every $\gamma \subset \theta_{n}^{t}$ there exists an $s_{\gamma}$ satisfying (16). After $n_{1}$ such steps, with $\theta / 2^{n_{1+1}}>\alpha_{1}$, all points of $Q-G_{0}$ will be included in the sets $1 A_{h}^{t}\left(h=0,1, \cdots, n_{1} ; t=1,2, \cdots, 2^{n_{1}}\right)$ (see (17)).

To avoid three indices let us enumerate the sets $1 A_{h}^{t}$ and their corresponding angles $\theta_{h}^{t}$ writing $1 A(i)$ and $1 \theta(i)$ with $i=1,2, \cdots, N_{1}$.

$k$ th step. Assuming that we have defined $(k-1) A(i),(k-1) \theta(i)$, $R_{k-1}$, and $E_{k-1}, i=1,2, \cdots, N_{k-1}$, we define $k A(i), k \theta(i), R_{k}$, and $E_{k}$ as follows: we cover p.p. the open kernel of $(k-1) A(i)$ (which has the same measure as $(k-1) A(i)$ as we can easily see) with a sequence $\left\{B\left(\epsilon / 2^{k-1} ;(k-1) \theta(i)\right)\right\}$ of disjoint sets similar to $B\left(\epsilon / 2^{k-1}\right.$; $(k-1) \theta(i))$ and similarly placed. The sets $S$ which are parts of the $B$ 's form another sequence $\left\{S\left(\epsilon / 2^{k-1} ;(k-1) \theta(i)\right)\right\}$ whose sum we denote by $E_{k}$; clearly

$$
\left|E_{k}\right|<\frac{\epsilon}{2^{k-1}}
$$

The subdivision of $Q$ in the first step into the sets $1 A_{h}^{\ell}$ we now perform on $(k-1) A(i)$ for every $i$, the only change being that instead of (16) we now must satisfy

$$
x \in s, \quad d s<\frac{1}{2^{k-1}}, \quad \frac{m_{1}\left(s \cdot E_{k-1}\right)}{m_{1}(s)}>\frac{1}{2} .
$$

We thus obtain the sets $k A(i)_{h}^{\ell}$ and the angles $k \theta(i)_{h}^{\ell}$; we continue this process until we cover all points of the $(k-1) A(i)$ except for a set of measure less than $\epsilon / 2^{k-1}$ (see (17)) which we include in an open set $G_{k}$ of measure less than $\epsilon / 2^{k-1}$; and with $R_{k}=E_{k}+G_{k}$ we have

$$
\left|R_{k}\right|<\frac{\epsilon}{2^{k-1}}+\frac{\epsilon}{2^{k-1}}=\frac{\epsilon}{2^{k-2}}
$$


The sets $k A(i)_{h}^{\ell}$ and $k \theta(i)_{h}^{\ell}$ we again enumerate for all $i, h$, and $t$, and we thus obtain $k A(i)$ and $k \theta(i)$.

We continue similarly. In this process the part of $Q$ which is not covered at least once by the sets $\{B\}$ has a zero measure; we cover it with a set $G$ of area smaller than $\epsilon$; with $E=G+\sum_{k=1}^{\infty} R_{k}$, we have $|E|<\epsilon+\sum_{k=1}^{\infty} \epsilon / 2^{k-2}=5 \epsilon$.

14. We shall now prove that $E$ satisfies (2B). Suppose $x \in Q$ if $x \in G_{k}+G$; then we have no problem since $x$ can be covered by a circle included in $E$.

Suppose then that $x \notin G_{k}+G$; then $x \in B\left(\epsilon / 2^{k-1} ;(k-1) \theta(i)\right)$ for a certain $i$ depending on $x$ (and $k$ ), and every $k$. From the way the different coverings have been performed, it follows that

$$
1 \theta(i) \supset 2 \theta(i) \supset \ldots \supset(k-1) \theta(i) \supset \ldots
$$

and since these angles are closed there must exist a direction $\boldsymbol{\gamma}_{x}$ included in all of them and such that (20) is true for every $k$ if the segment $s$ is parallel to $\gamma_{x}$; from that and the openness of $E,(2 B)$ follows easily.

The author is indebted to Professor A. S. Besicovitch for his many suggestions.

\section{REFERENCES}

1. H. Busemann and W. Feller, Zur Differentiation der Lebesgueschen Integrale, Fund. Math. vol. 22.

2. F. Riesz, Sur les points de densite au sens fort, Fund. Math. vol. 22.

University of Pennsylvania 